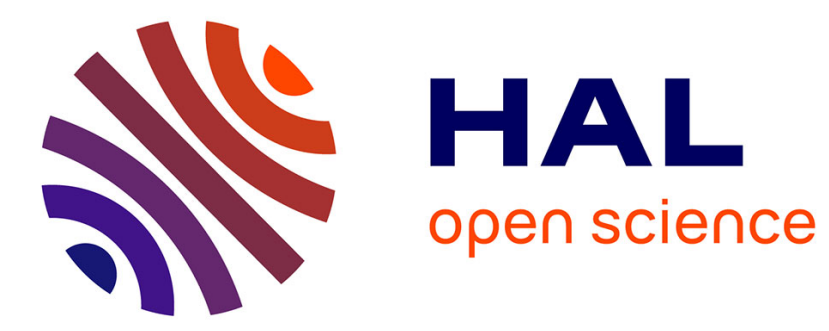

\title{
Du locus au territorium. Quelques remarques sur l'évolution des catégories en usage dans le classement des cartulaires méridionaux au XIIe siècle
}

Pierre Chastang

\section{- To cite this version:}

Pierre Chastang. Du locus au territorium. Quelques remarques sur l'évolution des catégories en usage dans le classement des cartulaires méridionaux au XIIe siècle. Annales du Midi : revue archéologique, historique et philologique de la France méridionale, 2007, 119 (260), pp.457-474. halshs-03364683

\section{HAL Id: halshs-03364683 \\ https://shs.hal.science/halshs-03364683}

Submitted on 4 Oct 2021

HAL is a multi-disciplinary open access archive for the deposit and dissemination of scientific research documents, whether they are published or not. The documents may come from teaching and research institutions in France or abroad, or from public or private research centers.
L'archive ouverte pluridisciplinaire HAL, est destinée au dépôt et à la diffusion de documents scientifiques de niveau recherche, publiés ou non, émanant des établissements d'enseignement et de recherche français ou étrangers, des laboratoires publics ou privés. 


\section{Du locus au territorium. Quelques remarques sur l'évolution des} catégories en usage dans le classement des cartulaires méridionaux au XIle siècle

\section{Pierre Chastang}

\section{Citer ce document / Cite this document :}

Chastang Pierre. Du locus au territorium. Quelques remarques sur l'évolution des catégories en usage dans le classement des cartulaires méridionaux au XIle siècle. In: Annales du Midi : revue archéologique, historique et philologique de la France méridionale, Tome 119, №260, 2007. Maîtrise et perception de l'espace dans le Languedoc médiéval. pp. 457-474;

doi : https://doi.org/10.3406/anami.2007.7196

https://www.persee.fr/doc/anami_0003-4398_2007_num_119_260_7196

Fichier pdf généré le 16/07/2018 


\section{Resumen}

Del locus al territorium. Observaciones sobre la evolución de las categorías utilizadas en la clasificación de los cartularios meridionales en el siglo XII.

Este artículo propone, mediante la comparación de dos cartularios del Bajo Languedoc redactados en el siglo XII y casi coetáneos, estudiar la evolución de los sistemas de clasificación topográfica utilizados en los medios eclesiásticos capitulares. Los últimos años del siglo XII se caracterizan por una doble evolución : es posible reconsiderar el papel de la iglesia como locus y el uso de los territoria derivados por aquel entonces del incastellamento. Esta evolución debe ponerse en relación con la difusión en los cabildos catedralicios de las summae en el código y en las institutes redactadas por los juristas meridionales. Estos textos, en los que se articulan la tradición jurídica romana y el discurso teológico sobre los lugares sagrados que remonta al siglo IX, permitirán la difusión de las categorías cultas entre los responsables de los textos escritos y su adaptación a la esfera de la escritura pragmática.

\section{Résumé}

Cet article propose, en comparant deux cartulaires bas-languedociens rédigés au XIle siècle à quelques décennies de distance, d'étudier l'évolution des systèmes de classement topographique en usage dans les milieux capitulaires. La fin du XIle siècle se caractérise par une double évolution : apparaît d'une part une réévaluation du rôle de l'église en tant que locus et l'utilisation des territoria récemment produits par l'incastellamento. Cette évolution doit être mise en relation avec la diffusion dans les chapitres cathédraux des Summae au Code et aux Institutes rédigées par des juristes méridionaux; ces textes, qui associent tradition juridique romaine et discours théologique sur les lieux sacrés élaboré depuis le IXe siècle, ouvrent la voie à la diffusion de catégories savantes vers les praticiens de l'écrit et à leur adaptation à la sphère de l'écriture pragmatique.

\section{Abstract}

From locus to territorium. Several remarks on the evolution of categories used in classifying southern cartularies in the twelfth century.

This article proposes, through the comparison of two cartularies from Lower Languedoc written in the twelfth century, several decades apart, to study the evolution of systems of topographical classification used in canonical chapters. The end of the twelfth century is marked by a double evolution : there appears a reevaluation of the role of the church as a locus, and the use of territoria recently defined through incastellamento. This evolution must be linked with the diffusion in cathedral chapters of Summae on the Code and on the Institutes written by southern jurists ; these texts, which associate Roman legal tradition and theological discourse concerning sacred places, elaborated since the ninth century, opened the way for the diffusion of learned categories towards those who used writing and to their adaptation to the sphere of utilitarian writing.

\section{Zusammenfassung}

Vom locus zum territorium. Bemerkungen zur Entwicklung des Klassizifizierungsvokabulars in den mittelalterlichen Kopialbüchern des 12. Jh.

In diesem Artikel untersucht der Autor am Beispiel zweier fast zeitgleicher Kopialbücher aus dem Niederlanguedoc wie sich die topographische Klassifizierung im 12. Jh. entwickelte. Am Ende des 12. Jh. wird die Rolle der Kirchen als locus wieder aufgewertet, anderseits werden die damals neuen territoria benützt, die durch das Phänomen des incastellamento entstanden waren. Diese dopppelte Entwicklung verläuft parallel in den Kanonikerstiften der Kathedralen mit der Verbreitung der Summae Codicis oder Institutionum, die von Juristen aus Südfrankreich verfasst wurden. In diesen Texten fusionieren juristische römische Tradition und theologischer Diskurs über heilige Stätten aus dem 9. Jh. Sie öffnen den Weg für die Verbreitung gelehrter Kategorien auch bei den Schreibern und ihre Anpassung an einen pragmatischen Schriftgebrauch. 
$\cap>$ 


\title{
Pierre CHASTANG*
}

\author{
DU LOCUS AU TERRITORIUM \\ QUELQUES REMARQUES \\ SUR L'ÉVOLUTION DES CATÉGORIES \\ EN USAGE DANS LE CLASSEMENT \\ DES CARTULAIRES MÉRIDIONAUX \\ AU XII SIÈCLE
}

Dans sa forme classique ${ }^{1}$, le cartulaire s'apparente au genre de la collection qui tient une place centrale dans la culture médiévale de l'écrit. Les préfaces et les dédicaces ${ }^{2}$ justifient souvent le travail de rassemblement des chartes par la mise à disposition des archives qu'il assure aux contemporains ${ }^{3}$. Les textes

*9, rue Vergniaud, 75013 Paris ; pierre.chastang@noos.fr

1. Je désigne ainsi les cartulaires qui, rassemblant privilèges et chartes privées, se diffusent dans l'ouest de l'Occident à partir de la seconde moitié du $\mathrm{XI}^{\mathrm{e}}$ siècle et qui remplissent une double fonction administrative et mémorielle ; voir DeCLERCQ (G.), « Originals and Cartularies : The Organization of Archival Memory (ninth-eleventh centuries) », Charters and the Use of the written Word in Medieval Society, K. HEIDECKER éd., Turnhout, 2000, p. 147-170, ici p. 167.

2. Voir le recensement des préfaces proposé par Pascale BouRgain et Marie-Clotilde HuberT, "Latin et rhétorique dans les préfaces de cartulaire », Les cartulaires. Actes de la table ronde organisée par l'École nationale des chartes et le GDR 21 du CNRS, PARISSE (M.), MORELLE (L.) et GuYotJeannin (O.) éd., Paris, 1993, p. 115-136.

3. Prenons trois exemples; les deux premiers concernent des codices monastiques de la première moitié du XII ${ }^{\mathrm{e}}$ siècle. Dans la préface du Tumbo A de la cathédrale de Santiago de Compostelle (1129-1134), la transcription des documents est assimilée à un rassemblement et une rénovation formelle des textes : «Bernaldus, prefate ecclesie thesaurarius, necessarium et utile aesse considerauit, quod illa omnia testamenta tranlatarentur et translata in uno libro, quasi in uno corpore, comprehenderentur, quatenus et illa que uestutatis consumcione iam litturata et delata erant, per translacionis nouitatem possent recuperari » (La documentación del Tumbo A de la catedral de Santiago de Compostela. Estudio y edición, LuCAs Álvarez (M.) éd., León, 1997,

(Suite de la note 3 page suivant)

Pierre Chastang est maître de conférences en Histoire à l'Université de Versailles-Saint-Quentin-en-Yvelines. Il est l'auteur d'une thèse dirigée par Monique Bourin qui a été publiée au CTHS en 2001 sous le titre : Lire, écrire, transcrire. Le travail des rédacteurs de cartulaires en Bas-Languedoc (XI'-XIII ${ }^{e}$ siècles). 
anciens sont retranscrits dans une écriture familière aux lecteurs et à cette rénovation matérielle s'ajoutent parfois, dans un continuum conduisant de la simple correction à la profonde réécriture, des transformations textuelles qui, désignées par les termes de reparatio ou de novitas, permettent de conformer l'héritage aux attentes présentes. Collection et assemblage de textes antérieurs, possédant une histoire propre, le cartulaire constitue un lieu d'observation fécond des mécanismes d'élaboration et de conservation documentaires. Partant du document dans sa matérialité, les fils de l'histoire particulière à chaque texte transcrit ayant été suivis, la logique sociale qui a prévalu lors de la mise en recueil des actes et de leur insertion dans une nouvelle intertextualité peut être restituée ; elle oblige à considérer l'écriture comme un processus dynamique qui participe à la production de la morphologie sociale médiévale ; en attestent les travaux récents sur les écritures communales italiennes ${ }^{4}$, les recherches consacrées au rôle de l'écriture, mémorielle et pragmatique, dans l'institutionnalisation de la vie des communautés et des ordres religieux ${ }^{5}$, ainsi qu'à sa contribution dans le processus historique d'invention des nations ${ }^{6}$.

\section{Suite de la note 3}

p.61-62). Une conception assez similaire est défendue par Pierre Diacre dans la dédicace à l'abbé Suniorectus qui ouvre son Registrum (1133) : «Unde quia usque in hodiernum res ipsa dilata est, uolo simul et jubeo ut priuilegia pontificum, præcepta imperatorum, regum, ducum, ac principum, nec non oblationes quorumcumque fidelium uno describantur uolumine » (Patrologie latine, vol. 173, col. 469-470). Dans la préface du cartulaire des Guilhem de Montpellier, la formulation prend un tour plus juridique et contentieux : « Hac agitur intentionis racione fuit voluntatis nostre propositum, ut omnia privilegia, omniaque alia instrumenta, que domini Montis pessulani possessionum causas determinant et exponunt, ad majorem diligentiam et evidentiorem cautelam, de confusionis sue multiplicitate in unum choartaremus volumen, ut, si quis deinceps possessionum ipsius ordinem turbare proponeret, statim e vicino confusus cognosceret quibus ipse titulis possidere gaudet » (Le cartulaire des Guilhem de Montpellier. Liber instrumentorum memorialium, Germain (A.-Ch.) et Chabaneau (C.) éd., Montpellier, 1884-1886, p. 2).

4. Citons, dans une bibliographie très fournie, le livre fondateur de CAmmarosano (P.), Italia medievale : struttura e geografie delle fonti scritte, Rome, 1991, le volume collectif Le scritture del comune : amministrazione e memoria nelle città dei secoli XII e XIII, ALBINI (G.) éd., Turin, 1998 (I florilegi, 12) et le livre Statutencodices des 13. Jahrhunderts als Zeugen pragmatischer Schriftlichkeit. Die Handschriften von Como, Lodi, Novara, Pavia und Voghera, Keller (H.), Busch (J. W.) éd., Munich, 1991 (Münstersche Mittelalter-Schriften, 64) qui constitue un bon exemple des travaux allemands dans ce domaine.

5. Voir, pour les travaux allemands, la présentation programmatique de Melville (G.), «Zur Funktion der Schriftlichkeit im institutionellen Gefüge mittelalterlicher Orden », Frühmittelalterliche Studien, 25, 1991, p. 391-417 ; voir également De ordine vitae : zu Normvorstellungen, Organisationsformen und Schriftgebrauch im mittelalterlichen Ordenswesen, Melville (G.) éd., Munich, 1996 et les travaux réalisés sur le thème de la mémoire des origines des ordres monastiques par Cécile Caby et Michel Lauwers (présentation du projet dans CABY (C.), « La mémoire des origines dans les institutions médiévales. Présentation d'un projet collectif », Mélanges de l'École française de Rome (Moyen Âge), 115, 2003, p. 133-140).

6. Voir, pour la Catalogne, Zimmermann (M.), Lire et écrire en Catalogne (IXe - XII siècle), 2 vol., Madrid, 2002 (Bibliothèque de la Casa de Velázquez, 23). 
La dimension historique et mémorielle des cartulaires a été privilégiée par l'historiographie ${ }^{7}$. Pourtant, ces derniers, en collationnant des chartes qui attestent de res localisées, constituent un outil pour comprendre, à l'échelle locale, la portée du moment «féodal » dans le processus médiéval de production de l'espace ${ }^{8}$, qui résulte d'une spatialisation du sacré ${ }^{9}$ décrite par Michel Lauwers comme « un lent et progressif inecclesiamento ${ }^{10}$. Se trouvent de la sorte profondément modifiés les liens associant l'ici-bas et l'au-delà ${ }^{11}$, l'articulation du local à l'universel au sein de l'Ecclesia, ainsi que les relations qui unissent l'Église aux laïcs.

Les auteurs des quelques articles de synthèse consacrés aux cartulaires ${ }^{12}$ opposent fréquemment deux grands types de codices, ceux qui classent les actes selon leur date et ceux qui, qualifiés de cartulaires topographiques, privilégient au contraire les lieux mentionnés. Cette dernière catégorie demeure cependant vague ; elle recouvre une grande variété de choix d'écriture et l'agencement topographique entretient bien souvent des liens étroits avec l'élaboration de la mémoire des institutions. Les dossiers hagiographiques, diplomatiques voire épiques concernant les origines des communautés monastiques, en abordant la question des lieux initiaux, de leur statut et de la territorialisation des pouvoirs exercés, proposent, par le récit, une jonction entre topographie et mémoire ${ }^{13}$.

7. Elle constitue le cœur de la démarche de P. J. GEARY, dont les travaux ont fortement contribué à faire sortir le cartulaire du strict champ de la diplomatique ; on pense à La mémoire et l'oubli à la fin du premier millénaire, Paris, 1996, mais avant cela à Aristocracy in Provence. The Rhône Basin at the Dawn of the Carolingian Age, Stuttgart, 1985 ; dans l'historiographie française, l'influence de Bernard Guenée et de ses disciples, au premier rang desquels J.-P. Genet, paraît primordiale (voir de ce dernier « Cartulaires, registres et histoire : l'exemple anglais », Le métier d'historien au Moyen Âge. Études sur l'historiographie médiévale, GuENÉE (B.) dir., Paris, 1977, p. 95-138).

8. Voir la synthèse suggestive proposée par J. BASCHET dans La civilisation féodale. De l'an mil à la colonisation de l'Amérique, Paris, 2004, p. 319-353.

9. Voir particulièrement LAUWERS (M.), Naissance du cimetière. Lieux sacrés et terre des morts dans l'Occident médiéval, Paris, 2005, Iogna-Prat (D.), La maison Dieu. Une histoire monumentale de l'Église au Moyen âge (v. 800-v. 1200), Paris, 2006 et À la recherche de légitimités chrétiennes. Représentations de l'espace et du temps dans l'Espagne médiévale (IX ${ }^{e}$-XIII ${ }^{e}$ siècle), HenRIET (P.) dir., Lyon, ENS éditions - Casa de Velázquez, 2003 (Annexes des Cahiers de linguistique et de civilisation hispaniques médiévales, 15).

10. Voir Lauwers (M.), Naissance du cimetière..., p. 269-276.

11. Voir les remarques de Guerreau (A.) : "Structure et évolution de la représentation de l'espace dans le haut Moyen Âge occidental », Uomo e spazio nell'alto medioevo, t. I, Spolète, 2003, p. 91-115.

12. Voir, pour les cartulaires anglais, Foulds (T.), « Medieval cartularies », Archives, XVIII, 77, 1987, p. 3-35 ; WALKER (D.), « The Organization of Material in medieval Cartularies », The Study of Medieval Records : Essay in Honour of Kathleen Major, Bullough (D. A.) et STOREY (R. L.) dir., Oxford, 1971, p. 132-150 ; DAvis (G. R. C.), Medieval Cartularies of Great Britain : A short Catalogue, Londres, 1958.

13. Voir Remensnyder (A.), « Topographies of Memory : Center and Periphery in High Medieval France », Medieval Concepts of the Past. Ritual, Memory, Historiography, AlthofF (G.), Fried (J.) et Geary (P.) éd., Cambridge, 2002, p. 193-214. 
À travers l'étude comparative du classement de deux cartulaires, nous aimerions évaluer les répercussions qu'ont pu avoir l'inecclesiamento et le développement, à partir de la seconde moitié du XI siècle, d'un discours ecclésiastique produisant une personnalisation de l'église ${ }^{14}$ sur les pratiques d'écriture dans les milieux canoniaux. Comment le discours savant qui a conduit à partir de la période carolingienne à ancrer l'Église en des lieux spécifiques a-t-il permis de produire des catégories efficientes dans la sphère de l'écriture pragmatique ? De quelle manière cette évolution s'accorde-t-elle avec l'incastellamento, phénomène majeur de l'histoire du peuplement durant cette période ${ }^{15}$ ?

Nous pensons que la relecture faite par les juristes du XII ${ }^{\mathrm{e}}$ siècle des textes du droit romain consacrés au statut des lieux sacralisés ${ }^{16}$ constitue un maillon essentiel dans la jonction qui s'opère alors entre la tradition juridique romaine et un discours théologique sur les lieux sacrés élaboré depuis le $\mathrm{IX}^{\mathrm{e}}$ siècle. Cette lecture ouvre également la voie à la diffusion de catégories savantes vers les praticiens de l'écrit. Comme l'a montré Yan Thomas, le droit romain objectivait « les trois catégories essentielles de la religion - le sacré, le religieux et le saint $-[\ldots]$ en trois statuts d'objets » et organisait « une projection spatiale de la limite à ne pas franchir $\gg^{17}$. La réviviscence du droit dans le milieu des chanoines du Midi confère à ces textes une importance et une efficacité nouvelles. Alors que les summae ${ }^{18}$ témoignent de la lecture qui fut faite des parties du corpus justinien consacrées aux lieux de culte, la pratique de cartularisation des milieux capitulaires atteste de l'usage pragmatique de ces catégories, en construisant, par l'écriture, un patrimoine conçu comme un espace structuré à partir des lieux consacrés et hiérarchisé par la propagation graduée du sacré. Elle permet à la fois d'affirmer les relations qui unissent le centre de l'institution aux périphéries qu'elle domine et d'évoquer le mode d'insertion de la « cellule » locale au sein de l'Église universelle.

14. Plusieurs textes témoignent de l'apparition de ce nouveau genre ; voir IogNA-PRAT (D.), La maison Dieu..., p. 325-362. Pour le Midi, voir en particulier la lettre du moine Garsia de Cuxa. Le texte est édité, traduit en castillan et commenté par TRIAS I TEIXIDOR (A.) dans « Espístola-sermón del monje de Cuixà », Anuario de fililogía (Barcelona), 1985, p. 19-47 ; voir également MuNDO (A. M.), «Recherches sur le traité du moine Garsias à l'abbé-évêque Oliva sur Cuxa », Cahiers de Saint-Michel de Cuxa, 1970, p. 63-74.

15. Voir Bourin (M.), Villages médiévaux en Bas-Languedoc. Genèse d'une sociabilité (Xe -

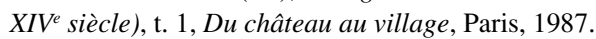

16. Sur les catégories antiques, voir DE SouZA (M.), La question de la tripartition des catégories du divin dans l'Antiquité romaine, Saint-Étienne, 2004.

17. Thomas (Y.), «Corpus aut ossa aut cineres », Micrologus, 7, 1999, p. 73-112.

18. Sur la production des summae méridionales, voir CORTESE (E), Il diritto nella storia medievale, t. II : Il basso medioevo Rome, 1995, p. 135 sqq. 


\section{Des lieux sans territoire : le cartulaire du chapitre cathédral de Nîmes}

Le milieu des chanoines, par son ouverture au droit ${ }^{19}$ comme par sa profonde implication dans le mouvement de réforme ecclésiastique ${ }^{20}$, constitue un lieu d'observation pertinent du renouveau de la culture et des pratiques de l'écrit au XII ${ }^{\mathrm{e}}$ siècle.

Le cartulaire du chapitre cathédral de Nîmes (Liber de honore canonicorum $^{21}$ ), qui a été rédigé peu de temps après $1117^{22}$ dans le contexte de la séparation des menses capitulaire et épiscopale consécutive à l'adoption de la règle de saint Augustin ${ }^{23}$, présente une grande homogénéité paléographique et codicologique. Actuellement constitué de dix quaternions et d'un ternion complets ainsi que de cinq cahiers lacunaires, le manuscrit contient un peu plus de 200 actes dont les dates s'échelonnent entre le $\mathrm{IX}^{\mathrm{e}}$ et le XII ${ }^{\mathrm{e}}$ siècle. Le cartulariste de Nîmes, dans son travail de transcription des documents du chartrier, a fait le choix d'un classement topographique dont la simplicité, qui ne peut être imputée à un manque de soin de sa part, contraste avec l'attention portée à la rédaction de rubriques conçues comme un véritable outil de recherche des textes. À cinq reprises, il actualise ainsi dans la rubrique des toponymes dont l'ancienneté risquait de rendre l'identification incertaine ${ }^{24}$.

Le rédacteur ne révèle qu'une partie de ses choix de classement ; le volume débute par un incipit (Incipit liber de honore canonicorum ${ }^{25}$ ) qui caractérise à la fois le contenu de l'ensemble du livre et, par opposition avec la partie

19. Parmi de très nombreuses études : Poly (J.-P.), « Les maîtres de Saint-Ruf, pratique et enseignement du droit dans la France méridionale au XII ${ }^{\mathrm{e}}$ siècle », Centre de recherches d'histoire institutionnelle et régionale, 2, 1978, Université de Bordeaux-I, p. 183-203. ; VONES-LIEBENSTEIN (U.), Saint-Ruf und Spanien...; et sur les chanoines de Nîmes, voir GouRON (A.), « Les chanoines de la cathédrale de Nîmes au milieu du XII ${ }^{\mathrm{e}}$ siècle : un milieu de chanoines lettrés ? ", Neuvième centenaire de la cathédrale de Nîmes (1096-1996), Nîmes 1997, p. 33-43.

20. Sur les liens entre droit et réforme, voir GIORDANENGo (G.), « Le droit romain au temps de la réforme : une étincelle ? (1050-1130) », Mélanges de l'École française de Rome - Moyen Âge, 113/2, 2001, p. 869-911.

21. Arch. dép. Gard, ms G 133 ; il existe une bonne édition de la fin du XIX ${ }^{\mathrm{e}}$ siècle : Cartulaire de l'église cathédrale Notre-Dame de Nîmes [désormais C.N.], GERMER-Durand (E.) éd., Nîmes, 1874.

22. Arch. dép. Gard, ms G 133, fol. 51 v = C.N., $\mathrm{n}^{\circ} 207$ (1117).

23. Pour le Languedoc, voir Élie GRIFFE (É), « La réforme canoniale en pays audois aux XI ${ }^{\mathrm{e}}$ et $\mathrm{XII}^{\mathrm{e}}$ siècles », Bulletin de littérature ecclésiastique de Toulouse, 44, 1943, p. 76-137 ; les deux volumes des Cahiers de Fanjeaux, n ${ }^{\circ} 2$ (La cathédrale [XII ${ }^{e}$ - XIV $V^{e}$ siècles], Toulouse, 1995) et 30 (Le monde des chanoines [XI ${ }^{e}$ - XIV $V^{e}$ siècles], Toulouse, 1989), et EsQUIEU (Y.), Autour de nos cathédrales. Quartiers canoniaux du sillon rhodanien et du littoral méditerranéen, Paris, 1992. Sur le cas spécifique du chapitre de Nîmes dans lequel la vie commune est attestée au début de la décennie 1070, voir CHASTANG (P.), Lire, écrire, transcrire. Le travail des rédacteurs de cartulaires en Bas-Languedoc (XI ${ }^{e}$ XIII ${ }^{e}$ siècles), Paris, 2001 (CTHS-histoire, 2), p. 221-238.

24. C.N., $\mathrm{n}^{\text {os }} 2$ (875-897), 21 (921), 23 (923), 36 (932), 90 (994).

25. Arch. dép. Gard, G 133, fol. 1. 
ouverte par un second incipit (Incipit de honore canonicorum qui extra civitatem continentur $^{26}$ ), les actes qui traitent de biens sis dans la cité de Nîmes ou dans ses environs immédiats. La seconde partie est consacrée au reste du comté et à quelques biens des comtés limitrophes.

Les dossiers ont donc été construits selon une logique globalement centrifuge qui souligne l'importance de la cité comme siège du pouvoir ecclésiastique, appliquant en quelque sorte à l'écriture du cartulaire, un topos qui, assez fréquemment présent dans un préambule de donation en usage dans le Midi, confond en un lieu - la civitas - et en une institution - la sainte église de Dieu -, la destination de l'aumône : Ad locum sanctae Dei ecclesiae, qui est situs infra Nemauso civitate, in honore sanctae Dei genetricis Mariae, sedem principalem, ubi x episcopus, preesse videtur... ${ }^{27}$. Pourtant, en l'absence d'évocation du modèle de l'Église primitive, médiatisé par les textes de réformateurs du $\mathrm{XI}^{\mathrm{e}}$ siècle ${ }^{28}$, le locus de Nîmes ne semble pas être, pour les chanoines, l'objet d'une ample profondeur mémorielle ${ }^{29}$. Cette opposition centre / périphérie est avant tout une manière de réserver une place centrale et ordonnatrice au lieu d'implantation des chanoines récemment régularisés, désormais contraints à la clôture.

\begin{tabular}{|l|c|c|c|}
\cline { 2 - 4 } \multicolumn{1}{c|}{} & Première partie & Deuxième partie & Ensemble \\
\hline Rubrique absente & 0 & 7 & 7 \\
Type d'acte & 0 & 1 & 1 \\
Type d'acte + anthroponyme & 0 & 2 & 2 \\
Anthroponyme & 18 & 15 & 33 \\
Obédience & 0 & 1 & 1 \\
Topographique & 27 & 137 & 164 \\
\hline
\end{tabular}

Types de rubriques présentes dans le C.N.

26. Arch. dép. Gard, G 133, fol. 24.

27. On le trouve dans 15 des 135 actes de donation copiés dans le cartulaire. Son utilisation est attestée de 911 jusqu'au début du XII ${ }^{\mathrm{e}}$ siècle.

28. Voir par exemple les références à saint Jérôme dans les bulles d'Urbain II confirmant la régularisation des chapitres méridionaux de Saint-Paul de Narbonne (hanc martyr et pontifex Urbanus instituit, hanc Augustinus suis regulis ordinavit, hanc Hieronymus suis epistolis informavit ; Gallia Christiana, VI, instr., col. 25-26), de Saint-Ruf (on retrouve la même phrase dans la bulle de 1092-99 ; Chevalier (U.), « Codex diplomaticus ordinis Sancti Rufi », Bulletin de la société archéologique de la Drôme, 25, 1891, p. 8-9) et de Maguelone (Gallia Christiana, VI, instr., col. 352-353.).

29. Sur la notion de locus médiéval, voir Zumthor (P.), La mesure du monde, Paris, 1993, p. 51-90. 
Les rubriques sont très majoritairement topographiques et le pourcentage est encore plus élevé lorsqu'il s'agit d'actes de cession de biens à l'Église. Le travail du rédacteur est largement tributaire des pratiques notariales en matière de localisation des biens. Trois systèmes dominent pour les biens situés en dehors de la cité de Nîmes :

1. Le plus fréquemment utilisé par les scribes est à trois niveaux : comté, villa et locus

2. Le second fait figurer la viguerie, intercalée entre le comté et la villa

3. Le troisième insère la mention du suburbium castri entre la viguerie et la villa.

Pour la localisation des biens situés dans la cité de Nîmes ou à proximité, deux types de rubriques dominent :

1. Le premier fait figurer la cité et le locus ou vicinium

2. Le second fait figurer le comté, la cité et le locus ou le vicinium.

\begin{tabular}{|c|c|c|c|}
\cline { 2 - 4 } \multicolumn{1}{c|}{} & Première partie & Deuxième partie & Total cartulaire \\
\hline Comté & & 1 & 1 \\
Viguerie & & 7 & 7 \\
Castrum/castellum & & 10 & 10 \\
Terminium & & 3 & 3 \\
Civitas & 15 & & 15 \\
Villa & 1 & 90 & 91 \\
Église & & 16 & 16 \\
Manse & & 1 & 1 \\
Locus & 10 & 9 & 19 \\
Vicinium & 1 & 137 & 164 \\
\hline Total & 27 & & \\
\hline
\end{tabular}

Types de lieux apparaissant dans les rubriques du C.N.

Dans les rubriques de la première partie, le rédacteur mentionne soit simplement la civitas, soit, pour d'évidentes exigences de précision, un ou plusieurs loci présents dans le texte. Pour les biens sis extra civitatem, le choix de la villa s'impose dans $84 \%$ des cas. À l'image de la civitas, la villa qui fonctionne comme un locus n'est jamais considérée dans sa dimension territoriale. Lorsque le bien est une église, la rubrique précise sa nature particulière, accompagnée du toponyme. Les seuls territoires qui servent à rubriquer sont l'Arisitum - ancien évêché du $\mathrm{VI}^{\mathrm{e}}$ siècle devenu viguerie - et la viguerie carolingienne de la Vaunage. Le rédacteur semble concevoir l'espace du diocèse comme formé d'une succession de loci et de «pays » plus vastes, dont la cohérence est moins institutionnelle que morphologique et humaine. C'est à cette même période, comme l'a montré Monique Bourin, que le système ortho- 
normé de description des parcelles disparaît, laissant le champ libre à la multiplication des mentions de lieux-dits ${ }^{30}$.

La cartographie de l'ordre de succession des cahiers permet de faire apparaître la logique de classement du manuscrit ${ }^{31}$. Aux biens situés à l'est et au sud de la cité succèdent les possessions occidentales puis septentrionales, ce qui est exactement l'ordre dans lequel les scribes de Nîmes énumèrent les confronts des parcelles dans les actes de la pratique ${ }^{32}$. Aucun territoire n'ordonne leur succession, mais l'ordre n'est en rien aléatoire. Les espaces scandés par les lieux mentionnés dans les rubriques du cartulaire ne se recoupent que rarement.

Ce système de classement se fonde sur une conception du locus proche de celle formulée par Isidore de Séville qui définit à partir du lieu initial directions, positions et rapport au temps : Locus est ubi sit, in foro, in platea. Loci autem motus partes sex habet, dextram, et sinistram, ante et retro, sursum atque deorsum. Parte quoque istae sex duo habent, id est situm et tempus. Situm, ut longue et prope. Tempus, ut heri, hodie. Porro situs a positione dictus, ut quis aut stet, aut sedeat, aut iaceat ${ }^{33}$. Le travail de classement des chartes transcrites s'organise en effet à partir d'un locus central, la civitas de Nîmes, et ce sont les formules en usage dans l'écriture des chartes qui semblent fournir au rédacteur du codex un modus operandi.

Ce classement des res ecclesiae à partir des partes d'un locus - ici la civitas - permet également de replacer, par emboîtement, le patrimoine local dans le cadre englobant de l'Église universelle. Ce lien est fréquemment exprimé sous une forme juridique dans les bulles de confirmation des patrimoines capitulaires qui, dans le contexte de développement de l'exemption ${ }^{34}$, rattachent les biens détenus au ius matris ecclesiae ${ }^{35}$. Aux res ecclesiae recensées s'appliquent les dispositions canoniques concernant la protection des patrimoines ecclésiastiques ${ }^{36}$, qui forment un ensemble d'autorités auquel les

30. BouRIN (M.), « Délimitation des parcelles et perception de l'espace en Bas-Languedoc aux $\mathrm{X}^{\mathrm{e}}$ et $\mathrm{XI}^{\mathrm{e}}$ siècles », Campagnes médiévales : l'homme et son espace. Études offertes à R. Fossier, Paris, 1995, p. 73-85.

31. Voir les cartes en annexes.

32. Voir note 30.

33. IsIdORE DE SÉviLle, Etymologiarum libri XX, LindSAY (W. M.) éd., Oxford, 1911, II, XXVI. Un exemplaire des Étymologies est mentionné dans l'inventaire de la bibliothèque du chapitre daté de 1219 .

34. Voir Falkenstein (L.), La papauté et les abbayes françaises aux XI ${ }^{e}-X I I^{e}$ siècles : exemption et protection apostolique, Paris, 1997.

35. Voir par exemple la bulle d'Urbain II en faveur du chapitre de Maguelone ; le texte précise que le patrimoine des chanoines ad ius matris ecclesiae pertineat (Gallia Christiana, VI, instr., col. 352-353).

36. Sur l'évolution de la législation canonique concernant la protection de la richesse temporelle de l'Église, voir Gilchrist (J.), «Eleventh and early Twelfth Century Canonical Collections and the Economic Policy of Gregory VII », Studi Gregoriani per la Storia de la "Libertas ecclesiae », 9, 1972, p. 378-417. 
dignitaires de l'Église se réfèrent pour trancher les conflits contemporains ${ }^{37}$. Si en 1120 , le lieu de culte ne joue pas encore un rôle structurant dans l'organisation du recensement patrimonial, le choix de la polarisation ecclésiale est clairement fait par Adrien IV $^{38}$ en $1156^{39}$ lorsqu'il confirme les biens du chapitre cathédral. Le texte, tout en reprenant le classement en deux parties du cartulaire, fait des églises l'unité de compte de l'énumération patrimoniale.

Le statut particulier de la civitas, centre géographique et institutionnel, explique la multiplication, dans la première partie du cartulaire, des actes qui ne sont pas rubriqués topographiquement, ainsi que l'ambiguïté de l'incipit. Des rubriques anthroponymiques coiffent en premier lieu des actes de donations, donations pro anima et donations testamentaires émanant de disposants importants - prêtres, abbés, chanoines, dignitaires du chapitre - avec, dans un certain nombre de cas, des services funéraires pesant sur le bien qui impliquent la conservation, par la communauté, des noms des bienfaiteurs. Des rubriques construites sur un modèle similaire ont été rédigées pour des actes accaptes, contrats ad edificandum / meliorandum, mises en tenure, déguerpissements - dans lesquels la potestas sur le bien a été transmise ou n'est pas totalement assurée. L'identification des personnes jouissant du bien s'avère essentielle à la bonne gestion patrimoniale. Des rubriques typologico-anthroponymiques introduisent des documents - chartes et censiers - qui, concernant des biens ou des revenus affectés à une dignité capitulaire particulière, révèlent l'organisation institutionnelle du chapitre.

Une lecture a-spatiale de certains actes a donc été privilégiée par le rédacteur lorsque ceux-ci abordent la question de la structuration institutionnelle du chapitre, des relations qui unissent les chanoines aux laïcs donateurs, singulièrement aux personnages issus des rangs de la noblesse locale.

37. John Gilchrist oppose à juste titre l'action sui iuris à celle secundum auctoritates qui n'implique pas une résolution des contradictions textuelles émanant de la tradition (« Eleventh and early Twelfth Century Canonical Collections... », p. 379).

38. Les liens tissés au cours de sa jeunesse par le souverain pontife avec les chanoines du Midi sont bien connus ; Nicolas Breakspear, futur Adrien IV, a fréquenté le milieu arlésien alors qu'il était chanoine (1140) puis abbé (1147) de l'ordre de Saint-Ruf ; voir DuCHESNE (L.), Liber pontificalis, t. II, p. 388 : hic namque pubertatis sue tempore, ut in literarum studio proficeret, egrediens de terra et de cognatione sua, pervenit Arelate, ubi dum in scolis vacaret, a Domino factum est ut ad ecclesiam beati Rufi accederet et in ea religionis habitum facta canonica professione susciperet. Proficiens ergo Deo auctore de bono semper in melius, prioratum in ipsa domo prius obtinuit, et postmodum ad abbatie apicem de communi fratum voluntate conscendit.

39. Arch. dép. Gard, G 133, fol. 45 à 46 v ( $8^{\mathrm{e}}$ cahier). La bulle est éditée dans C.N., n ${ }^{\circ} 213$ et Gallia christiana, t. VI, instr., col. 198. 


\section{Du locus au territoire : le cartulaire d'Agde}

L'organisation du premier cartulaire du chapitre cathédral d'Agde rédigé vers $1195^{40}$ présente deux évolutions notables par rapport au codex nîmois. Après un premier groupement d'actes consacré à la civitas, domine, dans la succession des dossiers, une logique centripète qui concourt à borner l'espace diocésain en suivant deux grands axes géographiques : la vallée de l'Hérault puis les bords de l'étang de Thau. Les actes transcrits forment ainsi neuf dossiers successifs qui concernent tour à tour les castra de Montagnac ${ }^{41}$ Aumes ${ }^{42}$, Touroulle ${ }^{43}$, Vias ${ }^{44}$, Bessan ${ }^{45}$, Fabricoles ${ }^{46}$, Mèze ${ }^{47}$, Florensac ${ }^{48}$, Marseillan ${ }^{49}-$ Caucs $^{50}$ et la villa de Pinet ${ }^{51}$.

Depuis 1150 , les centres paroissiaux ${ }^{52}$, jusqu'alors situés dans les villae, glissent vers les castra où se concentre la majorité de la population ; incastellamento et inecclesiamento se combinent pour fabriquer de nouveaux loci-lieux de culte et de vie -, points de stabilisation des rapports sociaux et des liens communautaires.

Par le classement adopté, les res ecclesiae se trouvent ainsi situées au sein de territoria. Il s'agit toujours, dans le cartulaire d'Agde, d'un système tacite qu'aucune rubrique ne vient dévoiler. Quoique décalée dans le temps, cette évolution paraît parallèle à l'effacement, dans les chartes du XII ${ }^{\mathrm{e}}$ siècle, de la mention du comté pour situer les biens, au profit du terminium du castrum, de la villa, ou de l'église.

40. Le manuscrit original, aujourd'hui disparu, a été copié par un érudit du XVIII ${ }^{\mathrm{e}}$ siècle nommé M. J. Gohin (Bibl. mun. de Montpellier, ms 33). Cette copie a été éditée par Raymonde FoREVILLE (Le cartulaire du chapitre Saint-Étienne d'Agde [désormais C.C.A.], Paris, CNRS, 1995) ; la première phase de compilation correspond aux 309 premiers documents édités.

41. Chef-1. de cant., Hérault.

42. Com. du cant. de Montagnac, Hérault.

43. Castrum auj disp., situé sur la com. de Bessan, cant. d'Agde, Hérault.

44. Com. du cant. d'Agde, Hérault.

45. Com. du cant. d'Agde, Hérault.

46. Castrum auj. disp., à identifier avec Saint-Martin (Agde) et l'église du Bagnas (Marseillan)

47. Chef-1. de cant., Hérault.

48. Chef-1. de cant., Hérault.

49. Com. du cant. d'Agde, Hérault.

50. Saint-Martin de Caucs, auj. sur le territ. de la com. de Mèze.

51. Com. du cant. de Florensac.

52. Citons deux exemples. Le premier concerne Touroulle : la première occurrence de l'église Saint-Laurent de Touroulle date de 1160 (C.C.A., $\mathrm{n}^{\circ}$ 37) et dès 1166 , une paroisse y est associée (C.C.A., $\left.\mathrm{n}^{\circ} 141\right)$, ainsi qu'un décimaire. Il coexiste jusqu'au XIII ${ }^{\mathrm{e}}$ siècle avec celui de SaintSylvestre de Mermian, villa voisine du castrum (C.C.A., $\left.\mathrm{n}^{\circ} 145\right)$. Le second exemple concerne Bessan : la première occurrence de l'église Saint-Pierre de Bessan date de 1166 (C.C.A., ${ }^{\circ}{ }^{\circ}$ 176) ; en 1187 ( $\mathrm{n}^{\circ} 141$ [1166]), elle apparaît comme paroissiale dans un testament (ecclesie sancti Petri de Beciano cuius parrochianus sum). 
Dans les dossiers du premier cartulaire d'Agde, la documentation « grégorienne »-j'entends par là les actes de restitution des dîmes et de droits ecclésiastiques - est regroupée et, la plupart du temps, placée en tête des dossiers concernant les biens patrimoniaux ordinaires. Les dossiers castraux sont donc scandés par les actes grégoriens. Le classement notifie ainsi la double importance des églises et des châteaux comme loci polarisant l'espace. Alors qu'un demi-siècle auparavant, le rédacteur du codex de Nîmes envisageait son travail archivistique comme un outil de défense et de recouvrement de l'ensemble des res ecclesiae ${ }^{53}$ organisé à partir de la civitas, son homologue agathois construit un classement territorialisé, polarisé par les castra et les églises.

L'usage du ressort castral dans l'écriture est sans doute imputable à l'origine sociale des chanoines, très largement issus des familles de la petite aristocratie locale. Mais la place nouvelle réservée au lieu de culte, comme locus d'où procède un principe d'organisation de l'espace, doit être liée à la diffusion dans le Midi de la France de summae exposant, en vue d'un usage pratique, les différentes parties du corpus justinien ${ }^{54}$. Ces textes produits, pour la plupart d'entre eux, dans la zone frontalière entre Languedoc et Provence ont connu une diffusion particulièrement intense dans un milieu canonial qui compte de nombreux juristes dont l'activité de conseil semble, à partir de la décennie 1160 , embarrasser l'autorité pontificale ${ }^{55}$. Nîmes constitue ainsi un des centres importants de développement des études juridiques et de production des textes. C'est la cité d'origine de Raimond des Arènes ${ }^{56}$, auteur des gloses Cardinalis des plus anciens manuscrits du Décret de Gratien ${ }^{57}$ qui est fait cardinal par Adrien IV

53. C'est la fonction assignée par Grégoire VII lui-même à la politique réformatrice : res ecclesiarum augmentare et defendere (Das Register Gregors VII, CASPAR (E.) éd., MGH Ep, Berlin, 1920-1923 [rééd. Munich, 1990], p. 33-36).

54. Pour le Midi, voir les volumes contenant les principaux articles de GouRON (A.) : La science du droit dans le Midi de la France au Moyen Âge, Londres, 1984 ; id., Études sur la diffusion des doctrines juridiques médiévales, Londres, 1987 ; id., Juristes et droits savants. Bologne et la France médiévale, Aldershot, 2000 ; id., Pionniers du droit occidental au Moyen Âge, Aldershot, 2006. Sur la chronologie de la redécouverte du Digeste, voir MüLLER (W. P.), « The Recovery of Justinian's Digest in the Middle Ages », Bulletin of Medieval Canon Law, 20, 1990, p. 1-29.

55. En 1168 ou 1169, Alexandre III défend aux chanoines d'exercer la fonction d'avocat en matière civile : ad audiendam nostram semel iterum pervenisse noscatis, quod quidam vestrum, peritam legum habentes, in causis secularibus, contra prohibitionem nostram et decretum etiam quod inde jam pridem edidimus, advocatione fungi presumunt, nec ab eis, sepe commoniti volunt aliqua ratione cessare (Bullaire de Maguelone, RouQuette (J.-B.) et Villemagne (A.) éd., t. 1, Montpellier, 1911, p. 151-152). Les mêmes interdictions sont reprises en 1186 ou 1187 par Urbain III, (ibid., p. 173-174). On retrouve cette interdiction au canon 12 du concile du $3^{\mathrm{e}}$ concile du Latran.

56. Voir Gouron (A.), « Le cardinal Raimond des Arènes : Cardinalis ? », Mélanges Gaudemet, Revue de droit canonique, 1978, p. 180-192 (repris dans La science du droit..., XII).

57. Sur la chronologie de rédaction du Décret et ses relations au corpus justinien, voir WINROTH (A.), « Les deux Gratien et le droit romain », Revue de droit canonique, 48/2, 1998, p. 285-299. 
en 1158 ; il l'avait fréquenté en Arles quelques années auparavant, dans le cadre de l'ordre des chanoines de Saint-Ruf ${ }^{58}$. C'est également à Nîmes qu'est écrite, dans ce même milieu, la collection canonique en 294 chapitres ${ }^{59}$ qui propose une synthèse entre traditions chartraine et italienne, mais dont le chapitre 241 dépend des Exceptiones legum Romanorum de Pierre ${ }^{60}$. La tradition manuscrite de la collection Caesaraugustana ${ }^{61}$, dont la première version doit être située vers Valence autour de 1120, est également liée au milieu rufien ${ }^{62}$.

Or, les contacts entre l'Agadès, le Nîmois ${ }^{63}$ et Arles sont intenses à partir de 1130 , date de l'accès au siège épiscopal d'Agde de Raimond de Montredon ${ }^{64}$, ancien chanoine de Nîmes et ancien archidiacre de Béziers. Les deux actes que Raimond de Montredon fait rédiger afin de procéder au partage des menses canoniale et épiscopale ${ }^{65}$ sont souscrits par des chanoines de Nîmes, dont Guiraud d'Esparron qui est témoin de la donation de l'église de Manduel aux chanoines de Saint-Ruf en $1106^{66}$.

Dans les années 1120-1140, un nouveau système d'autorités se met en place qui introduit une profonde rupture avec la sciencia canonica pratiquée dans les cloîtres $^{67}$. La coïncidence chronologique entre l'élaboration du classement du

58. Adrien qualifie ainsi Raimond de dilectus filius dans une lettre envoyée en 1157 à Saint-Ruf (Chevalier (U.), « Codex diplomaticus... », p. 37-38); voir également la note 38.

59. Texte inédit ; sur ce texte, voir Gouron (A.), « Sur la collection en 294 chapitres (ms de Prague Univ. VIII H.7 », Univ. de Bordeaux I. Annales de la faculté de droit, 2, 1978, p. 95-106 (repris dans La science du droit..., XIII) ; le lieu de rédaction de cette collection a été résolu par Poly (J.-P.), « Coheredes legum Romanorum. La renaissance du droit romain dans le Midi de la France », Historia del derecho romano. Trabajos en homenaje a Ferran Valls $i$ Taberner, t. X, p. 2923 , note 49.

60. Sur les textes et les manuscrits de la mouvance des Exceptiones Petri et de leur usage comme carrière de documents pour les romanistes, voir CORTESE (E.), Il diritto..., t. II, p. 47-55.

61. FouRNIER (P.), « La collection canonique dite Caesaraugustana », Revue d'histoire du droit français et étranger, 45,1921 , p. 53-79.

62. Le Bibl. nat. de France, ms lat. 3876 comme Barcelona, Arch. Cor. de Arag., ms San Cugat 63 contiennent le texte In die resurrectionis qui les associent au milieu rufien ; voir, FowLERMagerl (L.), « The Version of the Collectio Caesaraugustana in Barcelona », Ritual Text and Law. Studies in Medieval Canon Law and Liturgy presented to R. E. Reynolds, CusHING (K. G.) et GYNG (R. F.) éd., Aldershot-Burlington, 2004, p. 269-280.

63. Sur les compétences juridiques des chanoines de Nîmes, voir GouRON (A.), « Les chanoines de Nîmes au milieu du XII ${ }^{\mathrm{e}}$ siècle : un milieu de chanoines lettrés ? », Neuvième centenaire de la cathédrale de Nîmes (1096-1996), Nîmes, 1997, p. 33-43.

64. Il accède en 1143 à l'archevêché d'Arles.

65. C.C.A., $\mathrm{n}^{\circ} 10$ (1132) et $\mathrm{n}^{\circ} 11$ (1132) : in presentia dominorum <pour duorum $>$ canonicorum Nemausensium scilicet Guiraldi Despparone et Bertrandi de Leuco et in presentia duorum canonicorum Nemausensium scilicet Guiraldi de Sparrone et Bertrandi de Leuco. Dans le premier document, l'évêque réserve le ius consuetudinarium, ce qui est une nouveauté à cette date.

66. Chevalier (U.), « Codex diplomaticus... », p. 11-12.

67. Voir Giordanengo (G.), « Le droit romain au temps de la réforme... », p. 890 et id., «Auctoritates et auctores dans les collections canoniques (1050-1140) », Auctor et auctoritas. Invention et conformisme dans l'écriture médiévale, ZimMERMANN (M.) éd., École des chartes, 2001 (Mémoires et documents de l'École des chartes, 59), p. 99-129. 
cartulaire d'Agde et la mise en circulation de textes proposant les bases d'une réflexion renouvelée sur le statut des biens et la nature des lieux divins mérite d'être notée. Nous examinerons tour à tour cinq textes : la Summa institutionum Iustiniani est in hoc opere ${ }^{68}$, la Summa Trecensis ${ }^{69}$, Lo Codi $^{70}$, la Summa codicis ${ }^{71}$ de Roger $^{72}$ et les deux sommes de Placentin ${ }^{73}$ (Summa codicis ${ }^{74}$ et Summa institutionum $^{75}$ ).

La Summa institutionum Iustiniani est in hoc opere fut rédigée vers 1127 à Die, par un élève de Martinus $^{76}$. La mention dans le texte de Pons et d'Ugo

68. Summa institutionum "Iustiniani est in hoc opere »(Manuscrit Pierpont Morgan 903), LEGENDRE (P.) éd., Ius commune. Verröffentlichungen des Max-Plancks-Instituts für Europäische Rechtgeschichte, Frankfürt am Main, 1973.

69. Summa Codicis des Irnerius [= Summa Trecensis], FITTING (H.) éd., Berlin, 1894 ; voir Gouron (A.), « L'auteur et la patrie de la Summa Trecensis », Ius commune, 12, 1984, p. 1-38 (repris dans Études sur la diffusion..., III) et id., «L'élaboration de la Summa Trecensis », Sodalidas. Scritti in onore di Antonio Guarino, t. III, Naples, 1985, p. 3681-3696 (repris dans Études sur la diffusion..., IV). Le texte dans sa version primitive date de c. 1135 ; il a été remanié c.1140, puis rédigé de nouveau à la fin de cette même décennie, avant d'être complété c.1150. Il est désormais attribué à Géraud, maître originaire de Nîmes, d'Arles ou de Saint-Gilles. On lui attribue également le De natura actionum (Juristische Schriften des früheren Mittelalters, FitTing (H.) éd., Halle, 1876, p. 117-127).

70. La version occitane du texte est éditée sous le titre Lo codi. Eine Summa Codicis in provenzalischer Sprache aus dem XII Jahrhundert, DERRER (F.) éd., Zürich, Juris Verlag, 1974, ici p. 2-4 ; sur la dimension linguistique du plus long texte en prose occitane du XII ${ }^{\mathrm{e}}$ siècle, voir en dernier lieu GRAFSTRÖM (Å.), «Observations sur Lo Codi », Romania, 112, 1991, p. 155-186. La version en latin datée de 1173 est éditée sous le titre : Lo codi. Eine Summa in der lateinischen Übersetzung des Ricardus Pisanus, FitTing (H.) et Suchier (H.) éd., Halle, 1906, ici p. 1-4 ; sur l'attribution et la datation du texte, voir Gouron (A.), « Du nouveau sur Lo Codi », Tijdschrift voor Rechsgeschiedenis, 43, 1976, p. 271-277 (repris dans La science du droit..., VIII), et id., « L'auteur du Codi », Tijdschrift voor Rechsgeschiedenis, 70, 2002, p. 1-20. Le texte en occitan daterait de la fin de la décennie 1140, le premier manuscrit conservé (Sorbonne 632) a été rédigé c.1165; la première traduction en latin date des années 1158-1162, celle de Ricardus Pisanus de 1173. L'œuvre, rédigée à Saint-Gilles, serait attribuable à Raoul, chancelier du comte de Toulouse de 1155 à 1183.

71. Éditée dans Scripta anecdota glossatorum, t. I : Ugonis Sumuula de pugna, Rogerii Summa codicis, Questiones dominorum Bononiensium, Yrnerii formularium tabellionum, PALMERI (G. B.) éd., Bologne, 1888.

72. Sur ce personnage dont la biographie est obscure, voir Gouron (A.), « Rogerius, Quaestiones de juris subtilitatibus et pratique arlésienne : à propos d'une sentence archiépiscopale (1141, 5 novembre) », Mémoires de la société pour l'histoire du droit et des institutions des anciens pays bourguignons, comtois et romands, 34, 1977, Dijon, 1979, p. 35-50 (repris dans La science du droit..., XIV) ; l'attribution de ce texte d'origine padane, proposée par A. Gouron, est fortement critiquée. Roger, originaire d'Italie, a enseigné dans le Midi, peut-être à Montpellier, plus probablement à Arles.

73. Sur le personnage, voir Tourtoulon (P. de), Placentin, la vie, les auvres, Paris, 1896 et CORTESE (E.), Il diritto..., p. 138-143.

74. Placentini Summa codicis [reprint de l'éd. de 1536 avec l'édition du prologue], CALASso (Fr.) préf., Turin, 1962.

75. Placentini Summa institutionum [reprint de l'édition de 1535], Turin, 1973 (Corpus glossatorum juris civilis, 1).

76. L'auteur utilise quatre livres des Institutes, la première partie du Digeste, neuf livres du Code et pour les Novelles, il utilise systématiquement l'Épitomé de Julien bien qu'il connaisse les Authentiques. 
témoigne du lien qui existait entre le rédacteur de ce texte et le milieu des chanoines de Saint-Ruf. Le texte se réfère à la catégorisation romaine du sacré telle qu'elle est exposée dans le premier titre (De rerum divisione) du deuxième livre des Institutes de Justinien. L'opposition entre res humane et res divine, elles-mêmes divisées en res sacre, religiose et sancte, est reprise, et c'est sur cette dernière catégorie qu'insiste l'auteur ; convaincu qu'elle est plus difficile à saisir que les deux précédentes, il en donne la définition suivante : sanctum est quod ab iniuria hominum defensum atque munitum est, reprenant ainsi les termes de Marcien figurant dans le Digeste $(1,8,8)$. Le statut de res nullius des res divine est clairement réaffirmé : quod divini iuris est id nullius in bonis est (Digeste, 1, 8, 1). Dans la Summa Trecensis, le résumé du Code Justinien I, 2 (De sacrosanctis ecclesiis, de rebus et privilegiis earum) conduit à rappeler le rôle de mère de la foi et de la religion catholique exercé par l'Église (ecclesia quidem mater fidei ac religionis constituta est ${ }^{77}$ ) qui justifie la reconnaissance d'un droit particulier in retentione, siue alienatione seu in exactione des res ecclesiae nécessaires à l'exercice du culte et aux desservants. Et de rappeler en particulier le droit de prescription singulier accordé à l'Église ${ }^{78}$.

Dans la compilation en occitan intitulée Lo Codi qui dépend assez étroitement de la Trecensis, la question juridique de la préservation des patrimoines ecclésiastiques, abordée en I, 2 (De la sancta gleisas e de lors priuilegis e totas lors causas), est liée à la défense du dogme trinitaire et de la foi, " dont le respect et la conservation incombent à tous les hommes ». Mais l'auteur ne parle plus de l'Église mais des églises qui sont définies comme maires de fe e de religion. Le texte précise que les causas del mun - comme les terres, vignes et maisons - sont nécessaires aux églises comme aux altres locs honorables, justifiant le régime juridique spécifique qui s'y attache. Le texte poursuit en proposant une véritable personnalisation de l'église, locus à partir duquel irradie le sacré et personne juridique à laquelle s'attachent des droits spécifiques ed en retener las soas causas ed en requerre lo seus dreigz et une surprenante prosopopée fait dire à l'église à propos de biens qu'elle est en droit de revendiquer : aquesta causa es mia.

La Summa codicis de Roger est une œuvre inachevée, dépendante de la Trecensis $^{79}$. L'articulation du lieu de culte et des res ecclesiae est encore plus clairement exprimée : il faut prendre garde, nous dit le texte, que les privilèges accordés aux églises les englobent ainsi qu'ils englobent les biens qui en dépendent ${ }^{80}$, définissant ainsi une étendue sur laquelle s'appliquent des droits

77. L'expression n'est pas présente dans le Code.

78. Voir GiRARD (P. F.), Manuel élémentaire de droit romain, Paris, 1929², p. 321-336

79. Seule la première partie de cette Summa, jusqu'en C. 4, 57, est attribuée à Roger ; la fin a été complétée avec le texte de la Summa Trecensis.

80. Videndum est etiam priuilegia sint tributa ecclesiis circa se et circa res proprias. 
singuliers, à commencer par l'asile ${ }^{81}$ créé par les constitutions impériales de 419-431 qui ont prescrit l'extension de la sanctitas au-delà de l'autel ${ }^{82}$. Cette protection, que le texte détaille en des termes communs, est liée quelques lignes plus loin à une première définition de l'ecclesia comme lieu (et hoc verum est, si accipiatur in ea significatione qui dicitur locus). Et Roger poursuit par la deuxième acception du terme, celle qui définit l'église comme un rassemblement ou une congrégation de fidèles (pro conventione vel congregatione fidelium) à laquelle il lie le caractère inaliénable de la propriété ecclésiastique. Les biens appartenant aux églises (res propriae) ne peuvent pas être aliénés, jouissent d'une prescription particulière et sont protégés des charges pesant sur les terres ordinaires (retinet etiam res immunes ab extraordinariis et sordidis muneribus). Si la Summa institutionum de Placentin n'apporte rien de véritablement nouveau, sa Summa codicis revient sur la question de l'usage au pluriel du terme d'église dans le titre et le justifie d'une manière fort intéressante : In primis quaeri potest quare de ecclesiis titulus inscribatur plurative et non singulariter. Responderi potest, quia sub hoc titulo, non tantum de ecclesia Romana agitur, sed de omnibus ecclesiis, quae sunt in toto orbe terrarum. À chaque lieu de culte qui jalonne le monde, démultipliant la présence de l'Ecclesia, s'attachent des privilèges et Placentin associe très précisément ce statut des églises à la célébration du ministère divin et au sacrifice eucharistique qui s'y déroulent (et quidem ecclesia, dicitur locus uenerabilis, in quo diuinum ministerium celebratur, eucharistia consecratur). La monumentalité de l'église paraît donc secondaire dans la constitution du lieu vénérable et Placentin rappelle que l'on doit nommer église même un lieu vide (locus иасииs), c'est-à-dire un lieu qui n'est pas entouré de murs (parietibus circumdatus), à condition que la messe y soit dite (in aliquo loco missa canitur).

Ces textes proposant une synthèse du corpus justinien destinée aux spécialistes du droit comme aux praticiens insistent donc, au sujet des églises et des res ecclesiae, sur trois points essentiels :

1. Le Codi comme la Summa codicis de Roger proposent une véritable personnalisation des églises auxquelles se rattachent des droits spécifiques étendus aux res ecclesiae. L'Église est présente en une pluralité de loci, essentiellement définis par la célébration du mystère eucharistique.

2. L'exercice des droits ecclésiastiques fonctionne dans un cadre spatialisé ; aux églises, définies comme des lieux spécifiques ordonnant le monde, se rattachent les res ecclesiae, biens patrimoniaux nécessaires à l'entretien des bâtiments comme des desservants.

81. Roger écrit : Circa se habet hoc privilegium quod nemo de ecclesia invitus debet extrahi.

82. Voir Ducloux (A.), Ad ecclesiam confugere. Naissance du droit d'asile dans les églises (IVe-milieu du $V^{e}$ s.), Paris, 1994, p. 207-236. 
3. Enfin, les summae apportent des précisions sur le lieu de culte. L'église est à la fois un locus auquel se rattachent droits et biens et le rassemblement de la communauté des fidèles qui fonde l'inaliénabilité du patrimoine ecclésiastique.

C'est dans le contexte de la diffusion de ces textes que l'on assiste à la réévaluation du rôle de l'église dans un cartulaire d'Agde pourtant classé par territorium castri. Les églises dont dépendent les dîmeries sont mises en valeur dans ce cadre territorial laïque. Cet emboîtement notifie les enjeux de la réforme grégorienne et souligne en même temps la double polarité ecclésiale et castrale

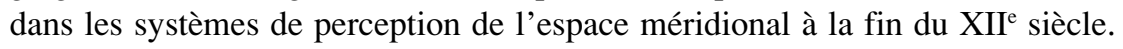
Mais les sommes méridionales au code et aux institutes témoignent avant tout d'une nouvelle juridicisation des catégories du sacré, prises en charge depuis l'époque carolingienne par un discours théologique et liturgique sur le lieu de culte. Cette évolution suscite un usage pragmatique de catégories désormais efficientes dans le travail de recensement, d'organisation et de défense du patrimoine ecclésiastique. Il faut attendre le $\mathrm{XIII}^{\mathrm{e}}$ siècle pour que la paroisse, précisément définie comme territoire dans la collection canonique d'Henri de Suse $^{83}$ serve, dans l'écriture des chartes, à la localisation des biens. Le rattachement des res ecclesiae à l'église et sa définition comme lieu de rassemblement des fidèles ont achevé de produire un territorium particulier.

83. HENRI DE SUSE, Summa aurea, III, 35, Venise, 1574 : Quid sit parochia: Locus in quo degit populus alicui ecclesie deputatus, certis finibus limitatus, et accipitur hic parrochia quatenus spirituale ius ecclesie se extendit. 


\section{ANNEXES}

Cartes de localisation des biens cités dans les rubriques des actes du cartulaire de Nîmes.

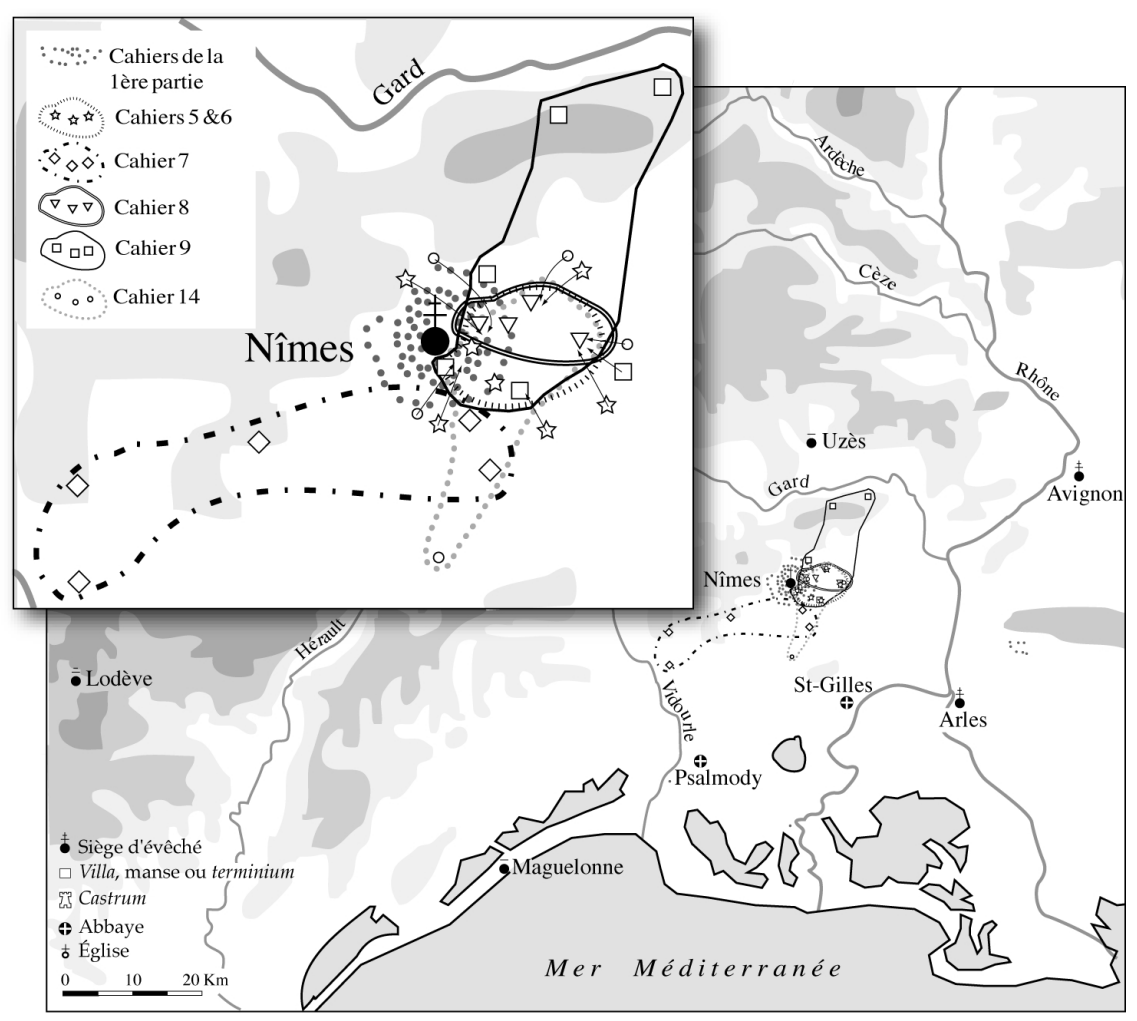

Localisation des biens cités dans les rubriques des actes de la première partie du codex et des cahiers 5 à 9 et 14 


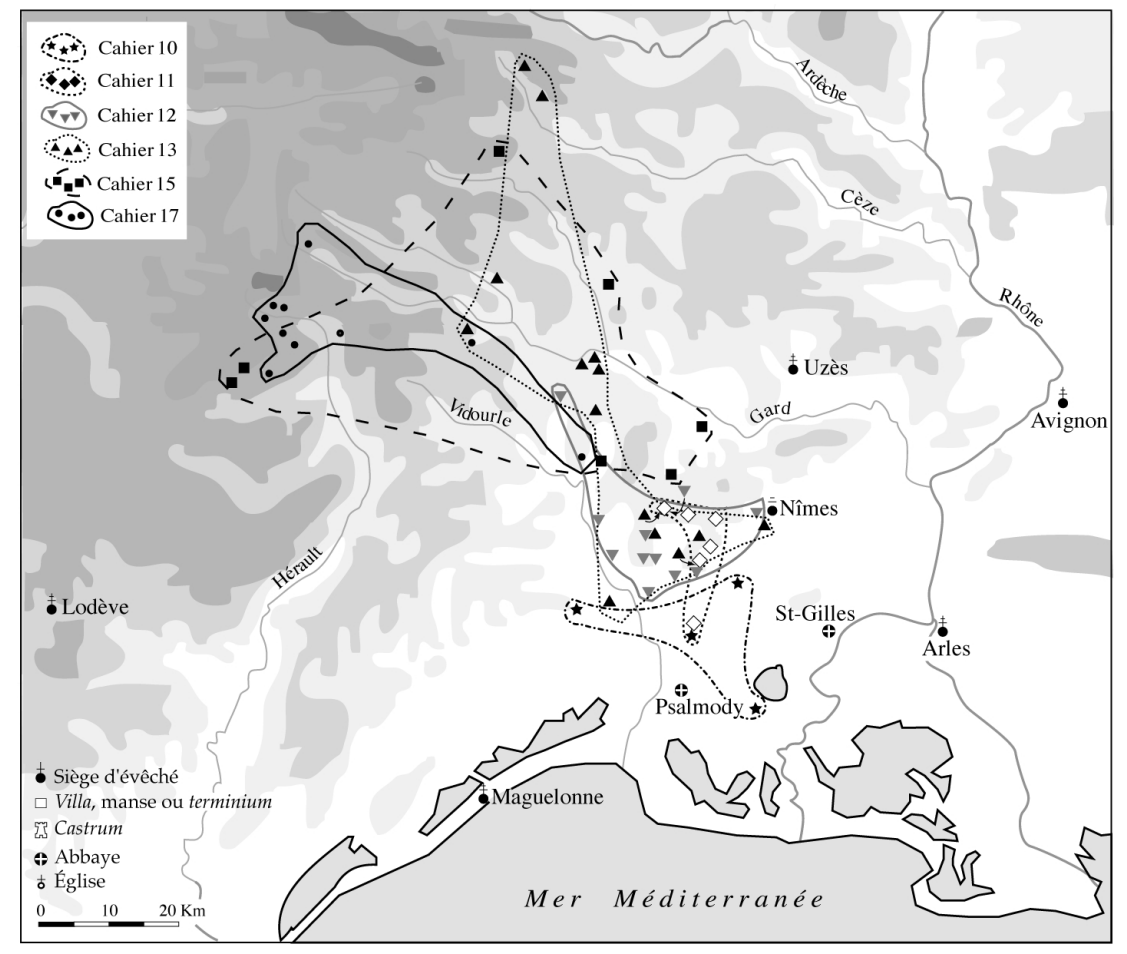

Localisation des biens cités dans les rubriques des actes des cahiers 10 à 13 et 15 à 17 